\title{
MODELING POLLUTANT TRANSPORT IN THE ATMOSPHERE BOUNDARY LAYER (U)
}

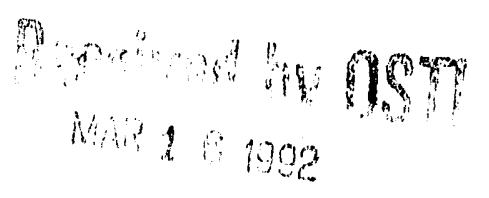

B. L. O'Steen

Westinghouse Savannah River Company Savannah River Site Aiken, SC 29808

\section{DISCLAIMER}

This report was prepared as an account of work sponsored by an agency of the United States Government. Neither the United States Government nor any agency thereof, nor any of their employees, makes any warranty, express or implied, or assumes any legal liability or responsibility for the accuracy, completeness, or usefulness of any information, apparatus, product, or process disclosed, or represents that its use would not infringe privately owned rig!ts. Reference herein to any specific commercial product, process, or service by trade name, trademark manufacturer, or otherwise does not necessarily constitute or imply its endorsement, recomand opinions for auth by the United States Government or any agency thereof. The views United States Government expressed kerein do not necessarily state or reflect those of the Government or any agency thereof.

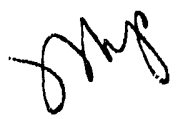




\section{SUMMARY}

The two basic methods for modeling the atmospheric transport of pollutants (diagnostic and prognostic) are examined along with the current models utilized at SRS for emergency response (WINDS). The ability of a limited-area (mesoscale) model, nested within a synoptic scale model, to represent a wide range of flow behavior, makes it the method of choice for predicting pollutant transport. Such a mesoscale model can provide an invaluable research tool and, with a periodic processing strategy for wind field calculation and/or sufficient computer capability, can be utilized in an emergency response capacity.

\section{INTRODUCTION}

In general, modeling the transport of an airborne contaminant requires knowledge of the 3-dimensional mean wind field, turbulent and convective motions which induce dispersion, and various mechanisms by which the contaminant is removed from the atmosphere. This information then serves as input to the conservation equation (along with source terms) for the spatial distribution of the contaminant concentration. The contaminant concentration and flux can then be used to evaluate radiological or other consequences of a pollutant release.

There are two basic approaches for solving the above transport problem:

(1) Calculate the mean wind field and turbulent/convective fluxes from the governing conservation equations given closure relations and appropriate boundary and initial conditions (prognostic).

(2) Measure the actual wind field and turbulence, and then input this information to the conservation equation (or assumed distribution) for the contaminant concentration (diagnostic).

In the first approach, one solves a set of Reynolds averaged conservation equations for mass, momentum, energy and water densities to obtain the mean wind field and, with suitable closure relationships, the turbulent fluxes of these densities. The primary feature of this approach is its inherent time dependent or prognostic capability. Thus extended forecasts of contaminant transport can be made. This is very important in an emergency response situation. Solving the full set of prognostic equations also allows terrain and other local effects to be taken into account. Numerical resolution will limit the ciegrec to which these effects can be represented, depending upon simulation domain and computational capability. Another distinguishing feature of the first approach is its large computational time requirement due to the complexity of the numerical solution techniques involved. However, real/CPU time ratios are currently much greater than unity and will certainly improve. The model domain considered here will be of limited extent, thus variation in the synoptic scalc conditions must be imposed through the top and lateral boundary conditions. Application of these boundary conditions is one of the more difficult problems associated with this approach since they are, in effect, artificial. Their interaction with outwardly propagating motions must be considered. Finally, the inherent uncertainty in the results of these 
calculations must be recognized. This uncertainty is due to an extreme sensitivity to initial conditions which is a consequence of the nonlin -ar nature of the equations. It affects not only forecast accuracy but also the ability to evaluate and/or compare different models. From a forecasting standpoint, one expects prognostic models to perform best when simulating stable,well-organized flows. The propagation of error (or lack of knowledge) in the initial conditions will be most rapid in fully developed, 3-D turbulent flow (Anthes et al., 1985).

The second approach, direct measurement of the wind field and turbulence, is possible since the wind field calculation is normally independent of the contaminant concentration. While this greatly simplifies the computational aspects of the problem, true forecasting capability is forfeited. However, persistence in meterological conditions generally makes the current wind field a good short term $(0-3 \mathrm{hr})$ predictor of the future wind field. Thus for highly turbulent meterological conditions, simply measuring the wind field probably provides a short term forecast which is (within inherent uncertainty) as good as that provided by a prognostic model. Longer term forecasts must still rely on some sort of prognostic modeling results.

Sparse wind field dati can be treated in two ways: (1) The data can be averaged (spatial and tempora!) to obtain a single wind speed and direction. (2) The data can be interpo'ated to produce a 2 or 3-D wind field. Various constraints (e.g. nondivergencu, can also be imposed on the interpolated wind field. The degree to which local terrain effects impact the interpolated field will depend upon the amount, location and interpolation of the data (Dickerson, 1978).

\section{CURRENT ATMOSPHERIC TRANSPORT MODELS AT SRS}

Currently, the modeling of atmospheric transport at SRS (WINDS system) relies primarily upon the measured wind field as input to a Gaussian plume model (PUFF/PLUME) for the contaminant concentration (Garrett, 1981). For a short term release, the source is treated as instantaneous and the wind measurements are averaged to produce a single wind speed and direction for advecting the contaminant cloud. The averaged wind may change speed and direction with time, but is considered constant within the contaminant cloud. Dispersion of the cloud is treated as a 3-D Gaussian process (Lagrangian reference frame) with a variance which increases with downwind distance. The dependence of the variance on downwind distance (or time) is determined empirically and is generally not found to be simply proportional to distance (Brownian motion). Thus the Gaussian model should not be considered as the solution to a classical (Fickian) diffusion problem in a moving coordinate system, although it does appear to imply a gradient flux representation of the turbulent mass transfer (Pasquill, 1983). The rate at which the variance increases with distance is determined from measured variances in both the horizontal and vertical wind directions.

A continuous release is handled in a similar manner by assumming a uniform contaminant concentration in the downwind direction due to advection and a 2-D Gaussian distribution perpendicular to the wind direction. Once again, the variance in this distribution increases with downstream distance and is determined empirically. This is the plume model. Unlike the puff model, the wind speed and direction must remain constant in time. Thus it is of rather 
limited utility for extended forecasting. This limitation of the plume model can be overcome by modeling the plume as a sequence of Gaussian puffs and then employing an analysis similar to the puff model to advect and disperse each of the individual puffs (2DPUFF). This basic sequential model is further improved in 2DPUF by interpolating a 2-D wind field based on both local and regional data (Addis and O'Steen, 1990). Thus the individual Gaussian puffs do not necessarily have the same direction or speed at the same point in time.

The above Gaussian models are, of course, diagnostic or possibly short term prognostic (persistence) if only measured winds are available. As previously noted, an extended forecast requires some input from a predictive model. To produce a long term prediction of contaminant transport at SRS, wind specd and direction along with two turbulence variables are forecast with the aid of Model Output Statistics (MOS), a statistical product of the NWS (Glahn, 1972). MOS combines synoptic forecast results from the NWS's limited fine mesh model (LFM) and local climatology to produce linear regression equations for predicting the local wind and turbulence (Gilhousen, 1980). The LFM model is a large scale $(160 \mathrm{~km}$ grid) free air model. While the locally measured wind field is still heavily relied upon for short term prediction, the synoptic scale LFM input to the MOS forecast makes it a better predictive tool than persistence alone for long term predictions (> $3 \mathrm{hr}$.).

\section{MORE RIGOROUS DIAGNOSTIC MODELS}

More rigorous diagnostic models generally perform a numerical integration of the 3-D contaminant conservation equation (advection-diffusion). This distinguishes them from the Gaussian puff-plume models. While a gradient representation of the turbulent flux is still normally assumed, the eddy diffusivity can now be made to reflect the vertical structure of the atmosphere (O'Brien, 1970). The required 3-D wind field is derived from the measured wind which has been interpolated to all points on the solution grid. The interpolated wind is usually adjusted to satisfy the constraint of a nondivergent velocity field since this is normally a condition imposed upon the contaminant transport equation. This constraint becomes more important as the complexity of the local terrain increases. The various diagnostic models in this more rigorous category are distinguished by the particular numerical solution technique ( finite-difference, finite elements, spectral, particle, etc) used to solve the advection-diffusion equation. Of course, these same solution methods can also be used in conjunction with prognostic wind models.

One such diagnostic model for pollutant transport is the LLNL code MATHEWADPIC. In this model, the pollutant transport equation is solved with a particle diffusion technique. Individual, distinguishable particles are moved on the grid according to a pseudo-transport velocity. This velocity consists of the local wind velocity plus a diffusion velocity due to gradients in the pollutant concentration as measured by the number of particles per grid cell. A nondivergent wind field is created (MATHEW) from measured winds for input to ADPIC. New particle coordinates are obtained from the old coordinates by a single forward difference in time, i.e. the solution scheme is explicit. The particle method has several advantages : (1) It is relatively fast, although for a given grid size the solution speed will depend on the number of particles present, (2) There is no numerical dispersion, and (3) Each particle can be tagged according to species, age, location, etc. There are two major drawbacks : 
(1) A large memory requirement and (2) Solution accuracy does not appear to be as good as that obtained by other methods. As an example, a boundary layer study with 3 pollutant species, 24000 cells and 30000 particles ran about 50 times faster than real time and required $90 \%$ of large core memory on a CDC 7600 machine. As will be seen later, this is 3 or 4 times faster than prognostic (mesoscale) models run on a similar machine.

MATHEW-ADPIC is but one example of this type of model or, more appropriately, solution method -. each with its own computational advantages and disadvantages. In terms of rigor, the primary advantage these methods enjoy over the various Gaussian models is relaxation of the Gaussian distribution constraint and the capability of handling nonuniform winds within the puff or plume.

\section{MESOSCALE ATMOSPHERIC MODELS}

In these models, the complete time dependent transport equations are solved for the mean wind field, turbulent fluxes, pressure, temperature and moisture content of the atmosphere. Typical spatial and temporal scales at which these equations are solved are 1 to 12 hours, $10 \mathrm{~km}$ to several hundred $\mathrm{km}$ horizontally and $10 \mathrm{~m}$ to $10 \mathrm{~km}$ vertically. Mesoscale models bridge the gap between the synoptic regime of 2-D flow and the microscale of fully developed 3-D turbulence. In the mesoscale model, atmospheric behavior at the microscale is described statistically and synoptic scale flow is either ignored (local forcing) or provides boundary conditions for the mesoscale model (synoptic forcing). Often these models are hydrostatic and the mass field is assumed to be anelastic or nondivergent to eliminate the propagation of sound waves. This is important since these high velocity sound waves control the solution stability. If the time-dependent mass continuity equation is retained, then the solution must be filtered to eliminate the high frequency sound waves or a very small time step must be used to insure instability (Pielke, 1984). The coordinate systems are usually $\mathbf{x}, \mathbf{y}$ and $\sigma$, where $\mathbf{x}$ and $\mathbf{y}$ represent various orthogonal projections onto a plane, and $\sigma$ is either a terrain following or isobaric vertical coordinate. Typical mesoscale grids vary from $1 \mathrm{~km}$ to $10 \mathrm{~km}$ horizontally and $10 \mathrm{~m}$ to $1 \mathrm{~km}$ vertically. Sub grid scale fluxes (turbulent and convective motions) are modelled by gradient relations (1st order closure) or can be calculated from prognostic equations subject to 2 nd order closure. Numerical solutions of mesoscale models are usually based on finite difference approximations to the differential equations. Although finite elements is generally considered state of the art in numerical solution methodology, it seems to have found limited acceptance among mesoscale modelers (Lee, 1977).

Boundary conditions can be a problematical feature in mesoscale modeling. The earth's surface is a real, physical boundary which interacts with the atmosphere through differential heating and variation in elevation. On the other hand, the top and lateral boundaries are artifical, and it is not always clear what constraints should be imposed. From the standpoint of boundary conditions, there are basically two types of mesoscale models: (1) Those which seek to model local forcing of the atmosphere (e.g. drainage flows and land-sea breezes) and thus attempt to minimize the effects of the lateral and top boundaries. (2) Those which seck to include the synoptic behavior at the boundaries. These require nesting of the mesoscale model within a synoptic scale model. If information passes only from the synoptic to the mesoscale, the 
nesting is said to be one-way interactive. If the mesoscale results are allowed to influence the synoptic scale model, then the nesting is said to be two-way interactive. While most mesoscale models have dealt with local forcing problems, the long range transport of contaminants will certainly require that synoptic scale flow be imposed in a one-way interactive scheme. An important aspect of this problem is devising a method of specifying synoptic boundary conditions so that the mesoscale forecast is influenced by the large scale forcing and yet not contaminated by erreneous reflection of energy from the boundary (Perkey, 1976).

As discussed in the Introduction, a major drawback to mesoscale modeling has been the computationally intensive nature of the numerical solution, i.e. large CPU times and storage requirements. In the following section, CPU times for several mesoscale model calculations are given. Assuming that a forecast for pollutant concentration at the SRS boundary is required within 5 minutes of a release, the indicated CPU speeds would be adequate if the calculations were performed on a CRAY. The same calculations could probably be performed rapidly enough on smaller and less expensive computers available today (e.g. a Stellar workstation) if a coarser grid spacing is acceptable. With continued development in computer technology, these calculations should eventually be practical on an inexpensive, desktop computer. Another possibility would be to make the wind field calculations in a periodic fashion with updated meteorological information and store the latest forecast for input to a pollutant dispersion code. If a release occured, it would only be necessary to run the dispersion code with the latest wind field result. Thus the forecast time would be limited to the time required to run the dispersion code, and the wind field calculation would not have to run at CRAY like speeds.

Finally, it should be recognized that the procedure(s) involved in making a diagnostic calculation will still be required to initialize the prognostic model. Thus the ability to make a rigorous diagnostic calculation will not be forfeited.

\section{EXAMPLES QF MESOSCALE MODELS}

In the following, several examples of mesoscale models are presented. The basic assumptions and computational features of each model are given. All except the last example represent models subject to local forcing. Only the LANL model actually incorporates the solution of a pollutant transport equation.

- LANL MODEL (Yamada, 1981)

Assumptions: Hydrostatic and incompressible

Coordinate System: UTM projection (horizontal) and terrain following (vertical)

Solution Method: Finite difference (ADI)

Initialization: Objective/Nondivergent

Turbulence: 2nd order 
Domain/Grid: $\quad \Delta x=\Delta y=380 \mathrm{~m}, \Delta z=4 \mathrm{~m}$ (expanding with height) on $10 \mathrm{~km} \times 7 \mathrm{~km} \times 1$ $\mathrm{km}$ domain. $\Delta \mathrm{x}=\Delta \mathrm{y}=10 \mathrm{~km}$ on $250 \mathrm{~km} \times 300 \mathrm{~km} \times 1-10 \mathrm{~km}$ domain

CPU Time: $0.4 \mathrm{~ms} /$ grid point/time step (CDC 7600)

Other: Pollutant transport equation solved by a particle method (Kao, 1988)

- NCAR MODEL (Clark, 1977)

Assumptions: Anelastic

Coordinate System: $x, y$ and terrain following (vertical)

Solution Method: Finite difference, Centered in time and space

Initialization: Combination dynamic and objective

Turbulence: 1 st order

Domain/Grid: $0.5 \mathrm{~km}<\Delta \mathrm{x}, \Delta \mathrm{y}<2 \mathrm{k}, 100 \mathrm{~m}<\Delta \mathrm{z}<500 \mathrm{~m} ; 50^{3}$ to $60^{3}$ grid points.

CPU Time: 0.05 to $0.1 \mathrm{~ms} /$ grid point/time step (NCAR Cray)

- COLORADO STATE MODEL (Pielke, 1974)

Assumptions: Hydrostatic and incompressible

Coordinate System: $x, y$ and terrain following (vertical)

Solution Method: Upstream spline interpolation for advection (with splitting); implicit diffusion approximation, centered in space for the pressure gradient force.

Initialization: Objective and short term dynamic

Turbulence: 1 st order

Domain/Grid: $\quad \Delta x=\Delta y=1$ and $10 \mathrm{~km}, \Delta z=2 \mathrm{~m}$ to $1 \mathrm{~km} ; 30 \times 36 \times 13$ grid points

CPU Time: $0.2 \mathrm{~ms} / g$ rid point/time step (NCAR Cray); approximately 24 minutes of CPU for a $12 \mathrm{hr}$. simulation

- WISCONSIN MODEL (Ballentine, 1980)

Assumptions: Hydrostatic

Coordinate System: $x, y$ and terrain following (vertical)

Solution Method: finite difference) Forward in time and upstream in space 
Initialization: Geostrophic wind from objective analysis

Turbulence: 1 st order

Domain/Grid: $\quad \Delta x=\Delta y=10 \mathrm{~km}, \Delta \mathrm{z}=50 \mathrm{~m}$ to $1 \mathrm{~km} ; 49 \times 41 \times 12$ grid points

CPU Time: NA

Other: Synoptic forcing, 2-way nested grid

\section{- ADVANCED REGIONAL ATMOSPHERIC MODELING SYSTEM (ARAMS)}

Assumptions: Bousssinesq, Quasi-compressible

Coordinate System: Horizontal -- cartesian or lat-lon

Vertical -- cartesian or terrain following

Solution Method: Finite difference; centered in space, combination leapfrog, pure forward, and forward-backward semi-implicit in time.

Initialization: Horizontally homogeneous, NMC data analyzed on isentropic surfaces and interpolated to model grid, NMC data interpolated to model grid.

Turbulence: Smagorinsky-type eddy viscosity, O'Brien profile funciion, Level 2.5 type closure with prognostic TKE equation.

Domain/Grid: $\quad 50 \mathrm{~m}<\Delta \mathrm{x}, \Delta \mathrm{y}<50 \mathrm{~km}, 1 \mathrm{~m}<\Delta \mathrm{z}<1 \mathrm{~km} ; 2$-way interactive grid nesting

CPU Time: Approximately $0.1 \mathrm{~ms} /$ grid point/ time-step (no cloud microphysics)

\section{CONCLUSICNS}

Clearly, mesoscale models represent the state of the art in limited area forecasting. Diagnostic models, while effective, have at most only short term forecasting capability and can only represent flow behavior which is actually measured. This is a serious drawback since the amount of data available is generally quite sparse. On the other hand, mesoscale models can incorporate the physics necessary to generate flow phenomena that is not observed in the initializing data. If problems related to synoptic forcing at the lateral boundaries can be dealt with, mesoscale model calculations along with large scale observations (as boundary conditions) should provide an operationally effective mettiod for making a detailed numerical forecast of meterological conditions. The major drawback to this approach is the rather large computer time required for the calculations. From a forecasting standpoint, these calculations are practical today with super computers such as the Cray. However, given the trend in computer technology, these calculations should 
become practical on less expensive machines in the near future. In the meantime, coarser solution grids and/or periodic calculation and storage of the wind field should aliow the mesoscale model to be used in an emergency response situation. 


\section{REFERENCES}

Anthes,R.A., Kuo,Y.H., Baumhefner,D.P., Enrico,R.M. and T.W. Bettge (1985), "Predictability of Mesoscale Atmospheric Motions", Adv. in Geophysics, 28B, 159.

Ballentine,R.J. (1980), "A Numerical Investigation of New England Coastal Frontogenesis", Mon. Weather Rev., 108,1479 - 1497.

Clark,T.L. (1977), "A Small Scale Dynamic Model Using a Terrain-Following Coordinate Transformation", J. Comput. Phys., 24, 186-215.

Dickerson, M.H. (1978), "A Mass Consistent Atmospheric Flux Model for Regions with Complex Terrain", J. Appl. Meteor., 17 (3), 241-253.

Garrett,A.J. and C.E. Murphy (1981), "A Puff-Plume Atmospheric Deposition Model for Use at SRP in Emergency Response Situations", USDOE Report DP-1595 Savannah River Laboratory, Aiken, S.C.

Gilhousen,D.B. and M.M. Pendergast (1980), "Automated Prediction of Tower Winds and Turbulence for the Savannah River Nuclear Facility", 2 nd Joint Conference on Applications of Air Pollution Meteorology, Mar. 24-27, 1980, New Orleans.

Glahn, H.R. and D.A.Lowry (1972), "The Use of Model Output Statistics (MOS) in Objective Weather Forecasting", J. Appl. Meteor.", 11, 1203-1211.

Kao, C-Y. J. and T. Yamada (1988), "Use of the CAPTEX Data for Evaluations of a Long-Range Transport Model with a Four-Dimensional Data Assimilation Technique", Mon. Weather Rev., 116, 293-306.

Lange, R. (1978), "ADPIC - A Three-Dimensional Particle-in-Cell Model for the Dispersal of Atmospheric Pollutants and Its Comparsion to Regional Tracer Studies", J. Appl. Meteor., 17, 312-319.

O'Brien, J.J. (1970), "A Note on the Vertical Structure of the Eddy Exchange Coefficient in the Planetary Boundary Layer", J. Atmo. Sci., 27, 1213-1215.

Pasquill, F. and F.B. Smith (1983), Atmospheric Diffusion, 3rd ed., Ellis Horwood Ltd, Chichester, England.

Perkey,D.J. and C.W. Kreitzberg (1976), "A Time-Dependent Lateral Boundary Scheme for Limited-Area Primitive Equation Models", Mon. Weather Rev., 104. 744.'755.

Pielke, R.A. (1974), "A 3-D Numerical Model of the Sea Breezes over South Florida", Mon. Weather Rev., 102, 115-139.

Pielke, R.A. (1984), Mesoscale Meteorological Modeling, Academic Press, Orlando, Fl.

Sherman, C.A. (1978) "A Mass-Consistent Model for Wind Fields over Complex Terrain", J. Appl. Meteor., 17, 312-319. 
Yamada, T. (1981) "A Numerical Simulation of Nocturnal Drainage Flow", $J$. Meteor. Soc. Jpn.", 59, 108-122. 

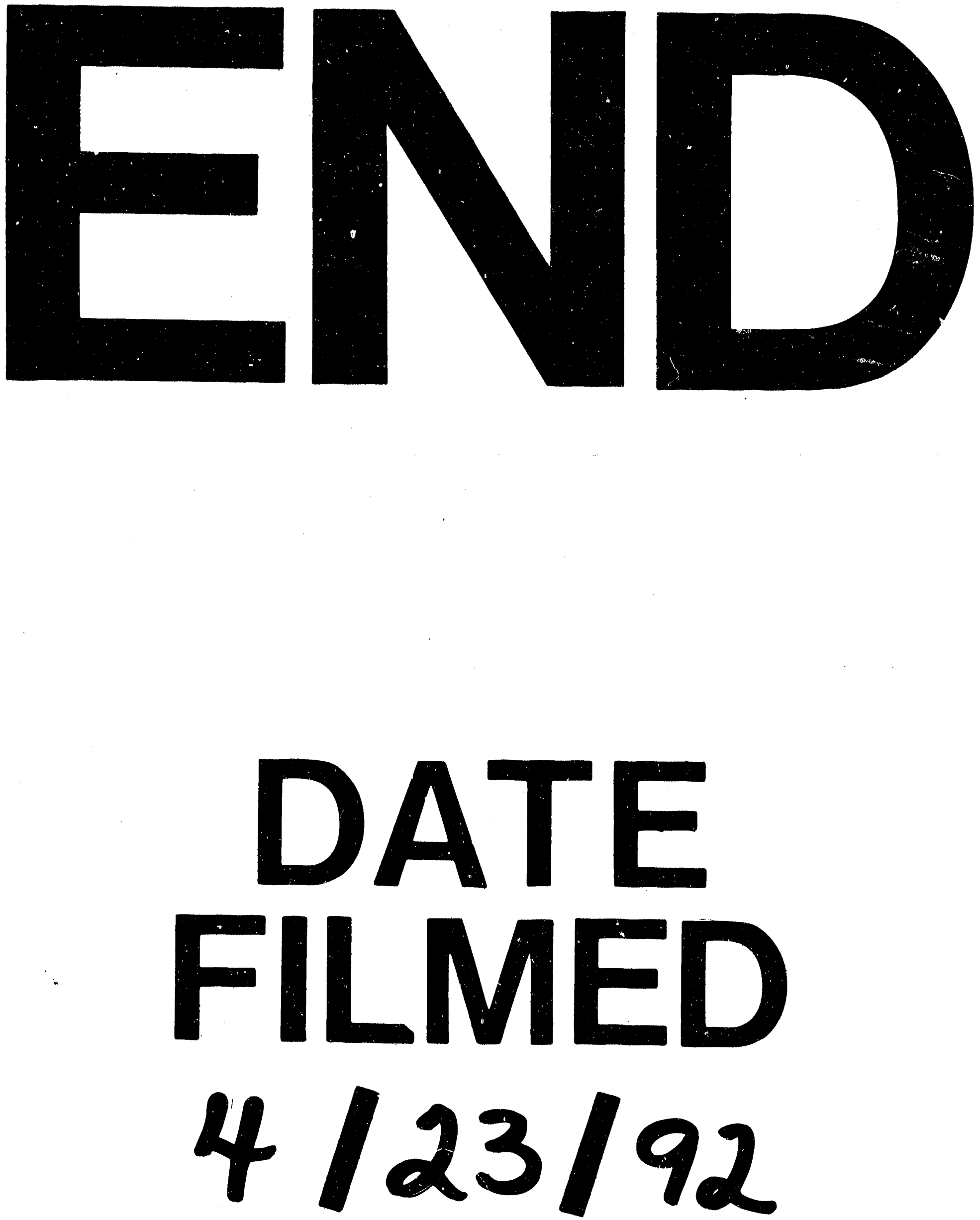

, 